\title{
PHYSICIAN-PATIENT COMMUNICATION REGARDING PRESCRIBED MEDICATION IN AN AMBULATORY CARE SETTING IN KATHMANDU, NEPAL
}

\author{
Joshi M P', Wachter D A ${ }^{2}$, Johnson K W', Regmi B $\mathbf{M}^{1}$ \\ Tamrakar $\mathbf{R} \mathbf{K}^{4}$, Ranjitkar $\mathrm{S}^{5}$, Lama $\mathbf{B}^{5}$, Sthapit $\mathbf{R}^{5}$, Dev $\mathbf{S}^{5}$, Subedi $\mathbf{S} \mathbf{R}^{5}$
}

\section{ABSTRACT}

Physician-patient interactions often lead to prescription of medicines. Safety and compliance in the use of these medicines are largely dependent on proper verbal as well as written communication between prescriber and patient. However, several published reports suggest that such communication is often inadequate. The present study indicated suboptimal doctor-patient communication at a tertiary care hospital in Nepal. Fifty-two (21.7\%) of the 240 patients/caregivers interviewed after out-patient consultation claimed that doctors did not provide any information on prescribed medicines. Nearly a quarter of the 188 patients/caregivers who did report having received information could not recall what they had been told, and in more than half of these cases the lack of recall was attributed to problems in communication. Frequent use of the English language and Latin abbreviation in prescribed dosing schedules indicated a need for improvement in written communication as well.

Key Words: prescribing information; communication; physician-patient interaction; Nepal.

1. Drug Information Unit, Dept. of Clinical Pharmacology, Institute of Medicine, Kathmandu, Nepal.

2. Dept. of Emergency Medicine, University of New Mexico School of Medicine, Albuquerque, NM, USA.

3. Drug Management Program, Management Sciences for Health, Arlington, VA, USA.

4. Pharmacist, Kathmandu, Nepal.

5. Pharmacy Assistant, Kathmandu, Nepal.

Address for correspondence : Prof. Mohan P. Joshi, Head of the Department of Clinical Pharmacology and Doctor-In-Charge of Drug Information Unit,

Maharajgunj Campus and T.U. Teaching Hospital

P.O. Box: 3578, Institute of Medicine, Maharajgunj, Kathmandu, Nepal. 


\section{INTRODUCTION}

Physician-patient communication regarding prescribed medication has been identified as an important determinant of medical compliance ${ }^{1-5}$ and the incidence of adverse drug outcomes., ${ }^{6,7}$ Although it is difficult to quantify physician-patient communication, studies have noted that patients are often dissatisfied with the information provided during consultation with physicians. ${ }^{7,8}$ Additionally, it has been documented that patients tend to have poor recall of drug-related information after leaving the hospital or physician's office..$^{8-11}$ These tendencies, reported in modern, industrialized countries, would be expected to be even more pronounced in underdeveloped regions where such problems as illiteracy, access barriers, and language barriers may be more common. The aim of the present study was to determine the extent of physician-patient communication regarding prescribed medication in an ambulatory care setting in Kathmandu, Nepal.

\section{METHODOLOGY}

Patients/caregivers were interviewed in the Bir Hospital compound after completing outpatient consultation, but before visiting a drug store or pharmacy. Bir Hospital is Nepal's oldest hospital, located in the center of Kathmandu. It is a 350bed tertiary care referral center and is associated with post-graduate medical teaching. Data were collected during a one-week period from July 27 to August 3, 1997. Prescribing doctors were unaware of the interviews.

A structured proforma was used to collect data from each patient/caregiver. A pretest was given to a total of 27 additional patients and the proforma was refined and finalized before use in the actual study. Some of the information collected in the proforma was copied from the original prescription and some was obtained from the patient/caregiver through the interview. The following patients/ caregivers were excluded from the study: those who were unwilling; health care personnel; those who were not prescribed any medicines; follow-up patients; and those with communication problems. Of the 291 patients approached, 240 were successfully interviewed. Of these 240 respondents, $221(92.1 \%)$ were the patients themselves and the remaining $19(7.9 \%)$ were caregivers accompanying children.

\section{RESULTS}

Diagnosis was mentioned in $124(51.7 \%)$ of the 240 prescriptions. There were a total of 475 drugs prescribed for the 240 patients, giving an average of two (range 1-4) drugs per prescription. Almost all $(98.7 \%)$ of the drugs were prescribed by brand names.

The written language in the majority of the prescriptions was English (93.3\%). The remaining $6.7 \%$ were in a combination of English and Nepali. For nearly a quarter $(22.5 \%)$ of all the drugs prescribed, the frequency of administration was written purely in Latin abbreviations. The prescribing doctor's signature was missing in 23 $(9.6 \%)$ of the prescriptions.

Patients/caregivers reported that the doctor gave information on the prescribed drugs in $188(78.3 \%)$ of the encounters; for the remaining 52 (21.7\%), patients/caregivers reported that no information was given at all. Of the 188 patients/caregivers who claimed to have received information on the dosing schedule of the prescribed drugs, 145 (77.1\%) recalled the information correctly while 43 (22.9\%) could not do so. When these 43 respondents were asked why they could not recall correctly, the reasons given were: forgot $(48.8 \%)$; information of technical nature $(23.3 \%)$; suggestion by doctor 
that detailed information would be given by the drug dispenser (14\%); noise in the outpatient department $(4.6 \%)$; time insufficient $(7.0 \%)$; and language problem $(2.3 \%)$.

Patients/caregivers claimed that before writing the prescription, the doctor asked about drug allergies in only $43(17.9 \%)$ cases and about concurrently taken medications in only $71(29.6 \%)$. Of the 240 patients, $79(32.9 \%)$ were married women of reproductive age (15-44 years). Only 11 (13.9\%) of these women claimed to have been asked if they were pregnant and only $4(5.1 \%)$ reported being asked if they were breast feeding.

\section{DISCUSSION}

Physician-patient communication regarding medication in our study sample was clearly suboptimal. Nearly a quarter of the patients interviewed claimed not to have been given any information at all about their prescriptions, and nearly a quarter of those who did report receiving information could not recall what it was they had been told. Much of this lack of recall may be attributed to poor communication on the part of the physician, including use of overly technical or indecipherable language, not devoting adequate time to explanation, or deferring the responsibility for providing detailed information to a drug dispenser. While drug sellers certainly have a responsibility to inform patients about dispensed medicines, physicians should at least be expected to provide basic information regarding the medicines they prescribe. This is particularly important in Kathmandu, where medicine shops are often staffed by personnel who are not formally trained in pharmacy, ${ }^{12}$ and whose practices have been shown to be suboptimal. ${ }^{13}$ It is also of concern that important queries into patient characteristics such as pregnancy, lactation, drug allergies and concurrent medication appear to have been largely omitted by physicians. This would represent a missed opportunity to educate patients about drug interactions and contraindications, and potentially result in exposure of patients to the risk of adverse outcomes.

Poor physician-patient communication has been described previously. In one patient recall-based study, only $58 \%$ of patients claimed to have received counseling on drugs by their physician, and only $30 \%$ remembered receiving warnings about potential pharmacotherapeutic risks. ${ }^{14}$ In another, less than one third of patients reported being given information on drug name, purpose, effects, and instructions for use. ${ }^{15}$ In a large telephone survey, just half of all patients claimed to have received information on the purpose of prescribed drugs or directions for their use, only $11 \%$ acknowledged receiving information on side effects, and 19\% reported that nothing at all was said about prescribed medication. ${ }^{16}$ In another series, even when interviewed immediately upon leaving the dispensary with the use of any available cues, only $58 \%$ of patients knew the dosing schedule of all prescribed medications, and only $65 \%$ knew the purpose of those medications. ${ }^{4}$ When videotaped, $86 \%$ of physicians were observed to have given instructions on how to take medications, yet only $33 \%$ addressed side effects. ${ }^{17}$

A large part of the communication problem may be attributable to a gap between the perceptions of physicians and patients. While $79 \%$ of one sample of physicians felt they spent enough time addressing medication issues, and $88 \%$ believed they informed patients adequately, fewer than $60 \%$ of their patients claimed to have been given any drug information, and only $2 \%$ described side effect counseling. ${ }^{18}$

Poor drug communication has been identified as one of the principal reasons why $30-55 \%$ of patients 
deviate from medical regimens. ${ }^{1,2,16}$ It has also been cited as a major factor in adverse drug outcomes. One study found a significant inverse relationship $(\mathrm{RR}=0.29)$ between drug knowledge/perceptions and such outcomes. ${ }^{6}$ Another, which critically examined audiotaped consultations identified misunderstandings in $80 \%$ of physician-patient interactions, all of which were linked to potential or actual adverse outcomes. ${ }^{7}$

To ensure healthy communication, patients must be willing to voice their concerns to physicians. However, even in developed countries, where such behavior has been encouraged over recent years, a significant problem persists. In one study, only $43 \%$ of patients who wanted a prescription and $20 \%$ who did not want one shared this preference with their physician; none of the patients who received unwanted prescriptions voiced their dissent. ${ }^{7}$ In a videotaped series of consultations, just $18 \%$ of patients expressed an opinion about a proposed drug prescription, and the physician sought an opinion in only $14 \% .{ }^{17}$ Another study in which a large number of patients were interviewed after physician encounters found that $26 \%$ of patients did not express their major concerns, chiefly because they felt no encouragement or opportunity. ${ }^{8}$ Especially in light of these results, it is clear that in a busy hospital in Nepal, where language and education barriers between physicians and patients are great, traditional and modern healing systems are at odds, and social etiquette mandates an attitude of quiet respect toward the physician, one could hardly expect to encounter the type of interchange necessary to create a mutually understood and acceptable treatment plan.

One final factor which must be considered in the attempt to characterize physician-patient communication is the accuracy of patient reporting. Several studies comparing patient reports and direct observation of the physician-patient encounter have documented poor patient recall of drug-related information, even moments after leaving the doctor's office. $^{8-11}$ Therefore, although we may conclude from our study that many patients were not well aware of their treatment regimen upon leaving the hospital, we cannot say with certainty that they were not well informed by their health care providers.

This study clearly demonstrates a problem in communication about medications between physicians and patients. As this study was limited to outpatient care at a single hospital, we cannot definitely extrapolate our results to the inpatient setting at Bir Hospital or services at other hospitals. However, it is likely that similar deficiencies exist more generally in Nepal's medical establishment.

As noted above, it is not possible at present to quantify the contribution of physician and patient factors. Further studies employing direct observation of the physician-patient interaction, while logistically difficult, could provide important information which might help guide recommendations to improve communication, and presumably patient comprehension and compliance.

In the mean time, it is important to ensure that detailed training in communication with patients be carried out across a broad range of specialties. Medical doctors, dentists, pharmacists, and other health care professionals need formal instruction in proper techniques of written as well as verbal communications as part of their professional training and their continuing education. Patients must be kept well informed of their treatment plans, using plain language and concepts which they can understand. If patients do not understand the details of their prescribed therapy, one may expect increased likelihood of dissatisfaction, noncompliance, and poor outcome. It is also important 
to educate the public more generally about basic concepts regarding the use of medicines and what they should expect from their health care providers. ${ }^{19}$ Although additional research may help refine specific educational initiatives, the need for such common-sense interventions cannot be denied.

\section{REFERENCES}

1. Kessler DA. Communicating with patients about their medications. New England Journal of Medicine 1991; 325(23): 1650-2.

2. Clark LT. Improving compliance and increasing control of hypertension: Needs of special hypertensive populations. American Heart Journal 1991; 121 (2 pt 2): 664-9.

3. McGrath JM. Physicians' perspectives on communicating prescription drug information. Qualitative Health Research 1999; 9(6): 731-45.

4. Fletcher SW, Fletcher R, Thomas DC, Hamann C. Patients' understanding of prescribed drugs. Journal of Community Health 1979; 4(3): 183-9.

5. Stewart JE, Martin JL. Correlates of patients' perceived and real knowledge of prescription directions. Contemporary Pharmacy Practice 1979; 2(3): 144-8.

6. O'Neil CK, Poirer TI. Impact of patient knowledge, patient-pharmacist relationship, and drug perceptions on adverse drug therapy outcomes. Pharmacotherapy 1998; 18(2): 333-40.

7. Britten N, Stevenson FA, Barry CA, Barber $\mathrm{N}$, Bradley CP. Misunderstandings in prescribing decisions in general practice: qualitative study. British Medical Journal 2000; 320(7233): 484-8.

8. Korsch BM, Negrete VF. Doctor-patient communication. Scientific American 1972; 227(2): 66-74.
9. Ley P, Spelman M. Communications in an outpatient setting. British Journal of Social and Clinical Psychology 1965; 4: 114.

10. Ley P. Primacy, rated importance, and the recall of medical statements. Journal of Health and Social Behavior 1972; 13: 311-37.

11. Joyce CR, Last JM, Weatherall M. Personal factors as a cause of differences in prescribing by general practitioners. British Journal of Preventive and Social Medicine 1968; 22(3): 170-7.

12. Joshi MP, Khakurel BK. Drug rationalization: now for the hard part. World Health Forum 1997; 18: 348-351.

13. Wachter DA, Joshi MP, Rimal B. Antibiotic dispensing by drug retailers in Kathmandu, Nepal. Tropical Medicine and International Health 1999; 4(11): 782-788.

14. Morris LA, Grossman R, Barkdoll GL, Gordon E. A national survey of prescription drug information provided to patients. Rockville, MD. Food and Drug Administration, 1986 (OPE study 73, May 1986).

15. Boreham P, Gibson D. The informative process in private medical consultations: a preliminary investigation. Social Science and Medicine 1978; 12(5A): 409-16.

16. Morris LA. A survey of patients' receipt of prescription drug information. Medical Care 1982; 20(6): 596-605.

17. Schofield TP. Patients should be given more information about drugs during consultations. British Medical Journal 1996; 313(7068): 1330.

18. Nightingale SL. Do physicians tell patients enough about prescription drugs? Postgraduate Medicine 1983; 74(2): 169-175.

19. Joshi MP, Onta S, Khakurel B, Bhattarai K. Rational drug use workshops for consumers in Nepal. Journal of the Institute of Medicine 1999; 21: 62-81. 